\title{
Structure of an ultrathin oxide film solved by machine learning enhanced global optimization
}

\author{
Lindsay R. Merte, ${ }^{1 *}$ Malthe Kjær Bisbo, ${ }^{2}$ Igor Sokolović, ${ }^{3}$ Martin Setvín,,${ }^{3,4}$ \\ Benjamin Hagman, ${ }^{5}$ Mikhail Shipilin, ${ }^{5}$ Michael Schmid, ${ }^{3}$ Ulrike Diebold, ${ }^{3}$ \\ Edvin Lundgren, ${ }^{5}$ Bjørk Hammer ${ }^{2 *}$
}

\author{
${ }^{1}$ Materials Science and Applied Mathematics, Malmö University; \\ 20506 Malmö, Sweden \\ ${ }^{2}$ Center for Interstellar Catalysis, Department of Physics and Astronomy, \\ Aarhus University; DK-8000 Aarhus, Denmark \\ ${ }^{3}$ Institute of Applied Physics, TU Wien; 1040 Vienna, Austria \\ ${ }^{4}$ Department of Surface and Plasma Science, Faculty of Mathematics and Physics, \\ Charles University; 18000 Prague 8, Czech Republic \\ ${ }^{5}$ Div. of Synchrotron Radiation Research, Lund University; \\ 22100 Lund, Sweden \\ *To whom correspondence should be addressed; \\ E-mail: lindsay.merte@mau.se, hammer@phys.au.dk
}

\begin{abstract}
Determination of the atomic structure of solid surfaces is a challenge that has resisted solution despite advancements in experimental methods. Theory-based global optimization has the potential to revolutionize the field by providing reliable structure models as the basis for interpretation of experiments and for prediction of material properties. So far, however, the approach has been limited by the combinatorial complexity and computational expense of sufficiently accurate energy estimation for surfaces. We demonstrate how an evolutionary algorithm, utilizing machine learning for accelerated energy estimation and diverse population generation, can be used to solve an unknown surface structure - the $(4 \times 4)$ surface oxide on $\mathrm{Pt}_{3} \mathrm{Sn}(111)$-based on limited experimental input. The algorithm is efficient and robust, and should be broadly applicable in surface studies, where it can replace manual, intuition based model generation.
\end{abstract}




\section{Introduction}

The atomic structure of surfaces and interfaces critically underpins our understanding of the performance of various materials, from heterogeneous catalysts and electrocatalysts to semiconductor electronics and photovoltaics. Despite the enormous importance of such fundamental information and the great advances in experimental instrumentation over the past decades, structure determination for complex surface reconstructions and ultrathin films remains a significant challenge, and many structures remain unsolved. Surface crystallography is labor intensive and prone to error, even when relatively simple structures are considered; complexity, disorder and heterogeneity can easily render such problems intractable. This naturally hinders our ability to interpret experiments that aim to establish structure-performance relationships for these materials, and especially our ability to predict and explain such relationships through quantum chemical modelling.

The oxidation of platinum-tin alloys provides a clear example of these limitations. Though the materials are of importance for a variety of catalytic processes, ${ }^{1-6}$ the structures of oxides formed on their surfaces and the details of metal-oxide interfaces involved remain poorly understood at the atomic level. This is despite several attempts, using a variety of techniques, to characterize experimentally the well-defined oxide layers formed on single crystal surfaces. ${ }^{7-12}$ A promising solution is to couple experiments closely with atomic structure prediction based on theory-driven global optimization. Density functional theory (DFT) based global optimization strategies have played an increasing role in structure prediction efforts since the early $1990 \mathrm{~s},{ }^{13}$ partly replacing labor intensive, intuition guided strategies. These optimization methods have gradually improved to handle increasingly large systems. Among others, ${ }^{14}$ evolutionary strategies $^{15-17}$ have been particularly successful on systems ranging from crystals to surfaces and nanoparticles, etc. ${ }^{18}$

Applicability to larger systems is, however, limited by the computational cost. This is most pronounced for systems involving a large number of atoms, as is the case for surfaces, which 
require inclusion of a certain number of bulk layers. ${ }^{19-21}$ As a solution, a new generation of structure prediction methods ${ }^{22-24}$ have achieved orders of magnitude reductions in computation time by partly replacing the expensive DFT potential with a machine-learned approximation refined during the search. Nevertheless, for many systems of interest, the cost of such computations remains high, and the exponentially-scaling complexity of the configuration space increases the risk that the global optimum structures are not found.

We demonstrate here the successful application of a machine learning enhanced algorithm to the problem of oxidized platinum-tin. Our approach makes use of the recently developed GOFEE algorithm, ${ }^{24}$ extended with a new and powerful technique for sampling the space of already identified structural candidates. The GOFEE method accomplishes a massive increase in efficiency while maintaining accuracy at the ab initio level. It incorporates, alongside planewave DFT, a machine-learned total energy surrogate model which is actively improved during an evolutionary search. This inexpensive model replaces DFT where high accuracy is not needed: in screening highly unstable candidate structures and in initial relaxation of structures toward local minima. Exploration of the configuration space is driven by uncertainty estimates from the surrogate model.

The extension of the method used here incorporates a feature based k-means clustering procedure in the population generation. Aside from one previous study, ${ }^{25}$ where clustering was employed to modify the fitness function used for deciding which population members to extract for offspring creation, we are not aware of other work applying clustering to improve population diversity in EA based atomistic structure search. Clustering has successfully been used to promote diversity in other fields. ${ }^{26-28}$ In these works, however, clustering was applied only to the very recent search history and much of the data accumulated during the search was thus left unused. The current approach, in contrast, retains the entire search history and thus utilizes all available data. This ensures structural diversity during the search, further improving reliability, and enables identification of the targeted surface structure among global optimum 

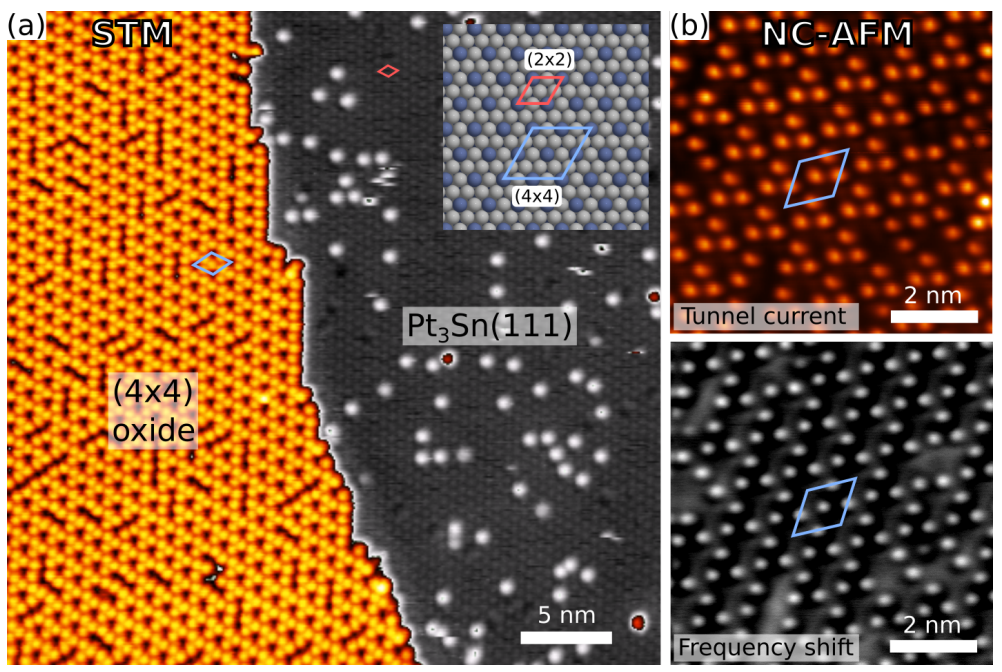

Figure 1: (a) Scanning tunneling micrograph showing the $(4 \times 4)$ oxide phase partially covering the $\mathrm{Pt}_{3} \mathrm{Sn}(111)$ surface. Inset is a ball model of the metal surface showing the unit cell dimensions of the alloy surface and the surface oxide. (b) Conductive non-contact atomic force micrographs of the $(4 \times 4)$ phase showing the tunnel current and frequency shift acquired simultaneously.

structures found for a range of compositions.

\section{Results}

The system under consideration here is the (111) surface of $\mathrm{Pt}_{3} \mathrm{Sn}$, the structure of which is shown in Fig. 1(a). This surface has been identified as having particular relevance for electrocatalytic reactions, ${ }^{29}$ and serves as a useful model for realistic materials. Oxidation of this surface results in formation of a well ordered oxide with $(4 \times 4)$ periodicity relative to the underlying face centered cubic metal lattice. Scanning probe microscope (SPM) images of the oxide (Fig. 1) show an array of protrusions, 3 per unit cell, in a chiral arrangement suggesting the symmetry of plane group $p 3$.

Attempts at structure determination for this phase have been made using several techniques, including scanning tunneling microscopy (STM), low energy electron diffraction (LEED), Xray photoelectron spectroscopy and diffraction (XPS/XPD) and low energy ion scattering (LEIS), ${ }^{10,11}$ 

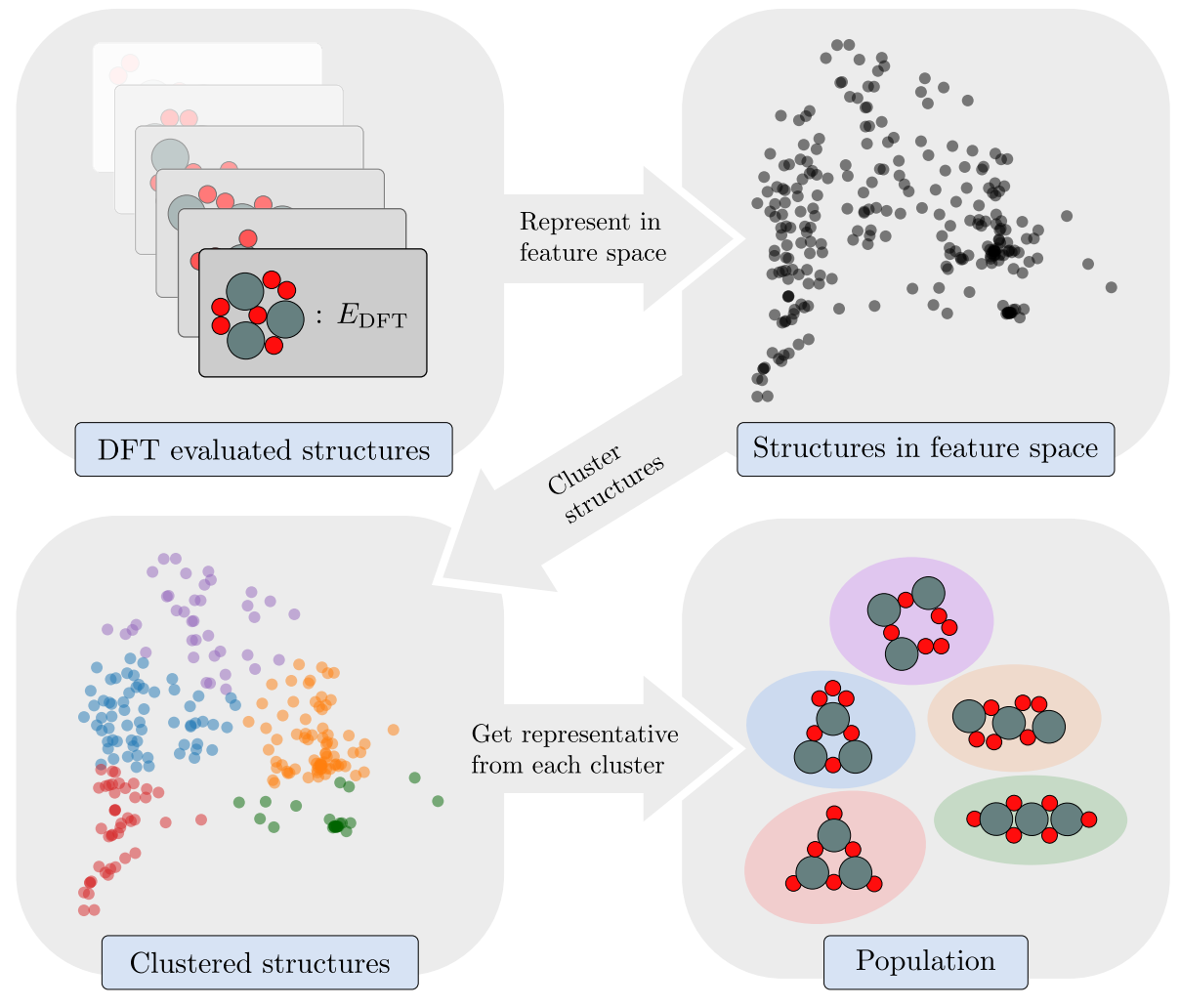

Figure 2: Sketch of the population scheme. For simplicity, data from a GOFEE search for 2D $\mathrm{Sn}_{3} \mathrm{O}_{6}$ clusters with $N_{\text {pop }}=5$ are shown. First, the current set of DFT evaluated structures are represented in a feature space and are subsequently clustered using the k-means++ algorithm, to identify groups of related structures. The population is then created by selecting one representative structure from each cluster.

and further attempts have been made to characterize very similar tin oxide phases formed by deposition and oxidation of $\mathrm{Sn}$ on $\mathrm{Pt}(111){ }^{7,9}$ Though it has been established that the structure is terminated by tin and oxygen, ${ }^{11}$ the tin in the structure shows an XPS binding energy very close to that of tin in the alloy, hindering characterization of tin in the oxide and leading to the suggestion that most of the tin in the structure is in fact still alloyed with platinum, in a so-called 'quasimetallic' state. ${ }^{9}$ The dominance of only three protruding atoms-of unknown type-per unit cell in scanning probe micrographs and the absence of other features that would guide the construction of atomic models has further hindered structure determination by direct deduction. 
As the composition of the $(4 \times 4)$ phase can not be deduced from experiment, the structure search algorithm must be applied to a range of $\mathrm{Sn}: \mathrm{O}$ combinations, and afterward subjected to thermodynamic analysis or comparison with experimental data to identify a likely candidate. The robustness of the search is therefore critical; the correct global minimum-energy structure must be identified for each composition if it is to be applied with confidence. In evolutionary algorithms, the concentration of members in the population with similar structural features can easily lead to premature convergence at a local minimum. This problem becomes increasingly pronounced for more complex systems, which exhibit many structurally diverse minima close in energy to the global minimum. The use of a machine-learned surrogate potential for candidate screening, as applied in GOFEE, increases the risk still further, as well-explored regions of the search space exhibit artificially lower predicted energies compared to the more conservative predictions in unexplored regions, thus reinforcing the bias toward structures that have already been tested.

The new population scheme which we have implemented to address this problem is depicted in Fig. 2, where we illustrate the approach with a $2 \mathrm{D} \mathrm{Sn}_{3} \mathrm{O}_{6}$ cluster. The population is generated from the full set of structures evaluated by DFT so far, after exclusion of those more than a fixed energy $\Delta E$ above the current lowest-energy structure. The remaining structures are represented in feature space and divided using the k-means++ clustering algorithm, ${ }^{30}$ which arranges them into families sharing similar characteristics. The population is generated by selecting the lowestenergy member of each family, promoting convergence to multiple local structural minima. ${ }^{31}$ In this way, the diversity of the population is maintained during the course of the search and sensitivity to the exploration diminishing effects inherent to the algorithm is reduced.

In our search for the structure of the $(4 \times 4)$ phase, we applied this algorithm using $\Delta E=$ $5 \mathrm{eV}$ and a population size $N_{p o p}=10$. We assumed a $(4 \times 4)$ periodic unit cell and a substrate consisting of bulklike $\mathrm{Pt}_{3} \mathrm{Sn}(111)$. The initial range of $\mathrm{Sn}: \mathrm{O}$ compositions selected for testing was based on the hypothesis that the $(4 \times 4)$ phase consists of a $\mathrm{Sn}^{2+}$ oxide monolayer of some 

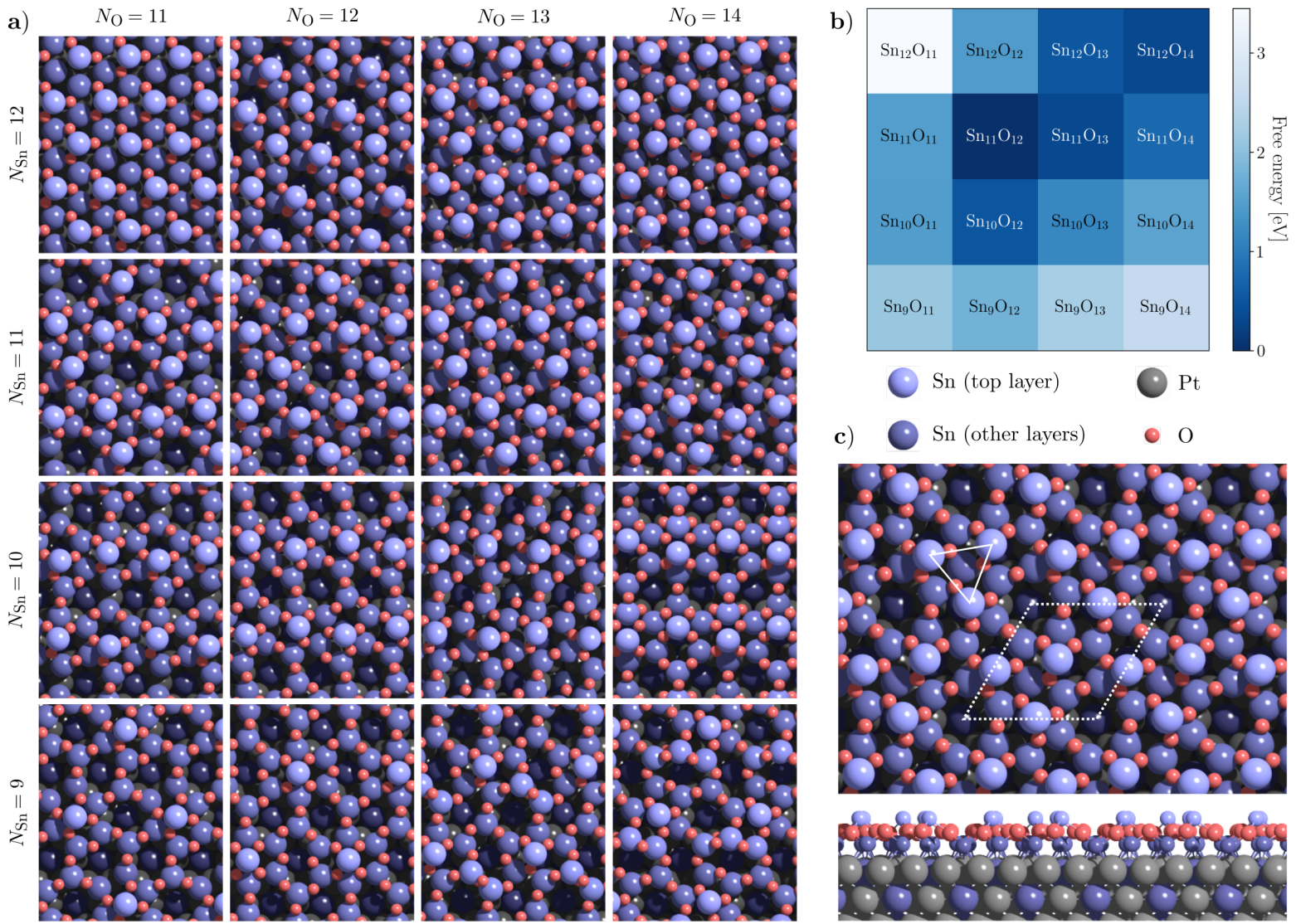

Figure 3: (a) Minimum energy structures found by the search algorithm for various compositions of $\mathrm{Sn}$ and $\mathrm{O}$ on $\mathrm{Pt}_{3} \mathrm{Sn}(111)$. (b) Calculated free energies for the different structures under the experimental conditions $\left(10^{-5}\right.$ mbar, $\left.600{ }^{\circ} \mathrm{C}\right)$, relative to the $\mathrm{Sn}_{11} \mathrm{O}_{12}$ structure. (c) Model of the lowest-energy $\mathrm{Sn}_{11} \mathrm{O}_{12}$ structure, corresponding to the observed $(4 \times 4)$ phase.

sort, taking inspiration from bulk $\mathrm{SnO}$ and $\mathrm{SnS}$, which are composed of van der Waals sheets, with $\mathrm{Sn}^{2+}$ in 4-fold and 3-fold coordination, respectively. Supported on $\mathrm{Pt}_{3} \mathrm{Sn}(111)$, such sheets would exhibit tin densities between 8 and 13 atoms per $(4 \times 4)$ unit cell. With this density range in mind and supposing somewhat higher coverages of oxygen compared to tin due to presumed oxygen affinity of tin in the substrate, we selected 16 combinations, with 9-12 Sn and 11-14 O per cell.

The search algorithm was then executed for all of these compositions and repeated three times from independent starting configurations to check for consistency. For all but three com- 
positions $\left(\mathrm{Sn}_{9} \mathrm{O}_{11}, \mathrm{Sn}_{9} \mathrm{O}_{13}\right.$ and $\left.\mathrm{Sn}_{9} \mathrm{O}_{14}\right)$, the same minimum-energy structures were found in all four runs of the search. The lowest-energy structures found are depicted in Fig. 3. Most of the structures found exhibit a common feature: a minority of $\mathrm{Sn}$ atoms protrude from the surface and show a lateral spacing of $\sim 5-6 \AA$, consistent with SPM images of the $(4 \times 4)$ and related phases. The remaining $\mathrm{Sn}$ atoms are at the metal interface, with $\mathrm{O}$ atoms forming a layer in between. One of the 16 structures found exhibits the $p 3$ symmetry expected from experiment: that with a composition $\mathrm{Sn}_{11} \mathrm{O}_{12}$, with three protruding $\mathrm{Sn}$ atoms, $8 \mathrm{Sn}$ atoms at the interface, and 12 nearly coplanar $\mathrm{O}$ atoms in between. Estimates of the free energies of the different structures under the conditions of the experiment also indicate that the phase is the most stable (Fig. 3b).

Surface X-ray diffraction (SXRD) confirms this structure and enables further refinement. Fig. 4(a) shows fits to measured rod profiles following structure refinement with the theorybased $\mathrm{Sn}_{11} \mathrm{O}_{12}$ structure as the starting point. The fits to the experimental rod profiles are excellent, and the in-plane structure factors calculated for the relaxed model reproduce the experimental ones as well (Fig. 4(b)). During refinement, the overlayer atoms moved toward the surface by $0.1-0.2 \AA$, consistent with the well-known underbinding of the employed DFT functional, but the final structure is otherwise nearly identical to that produced by the search algorithm. Simulated AFM images for the structure (Fig. 4(c)) also show good agreement with experiment, exhibiting the characteristic chiral pattern with 3 protrusions per unit cell, corresponding to the three protruding $\mathrm{Sn}$ atoms in the structure.

\section{Discussion}

The $(4 \times 4)$ phase can be described as a $\mathrm{Sn}^{2+}$ surface oxide-a contiguous two-dimensional network related to $\mathrm{SnO}$, but with a structure strongly influenced by bonding with the metal substrate. The basic structural element is $\mathrm{Sn}$ in 3-fold oxygen coordination, with a strongly asymmetrical pyramidal geometry. This geometry differs somewhat from that found in bulk $\mathrm{SnO}$, a layered material composed of buckled $\mathrm{SnO}$ sheets where $\mathrm{Sn}$ adopts a 4-fold pyramidal 

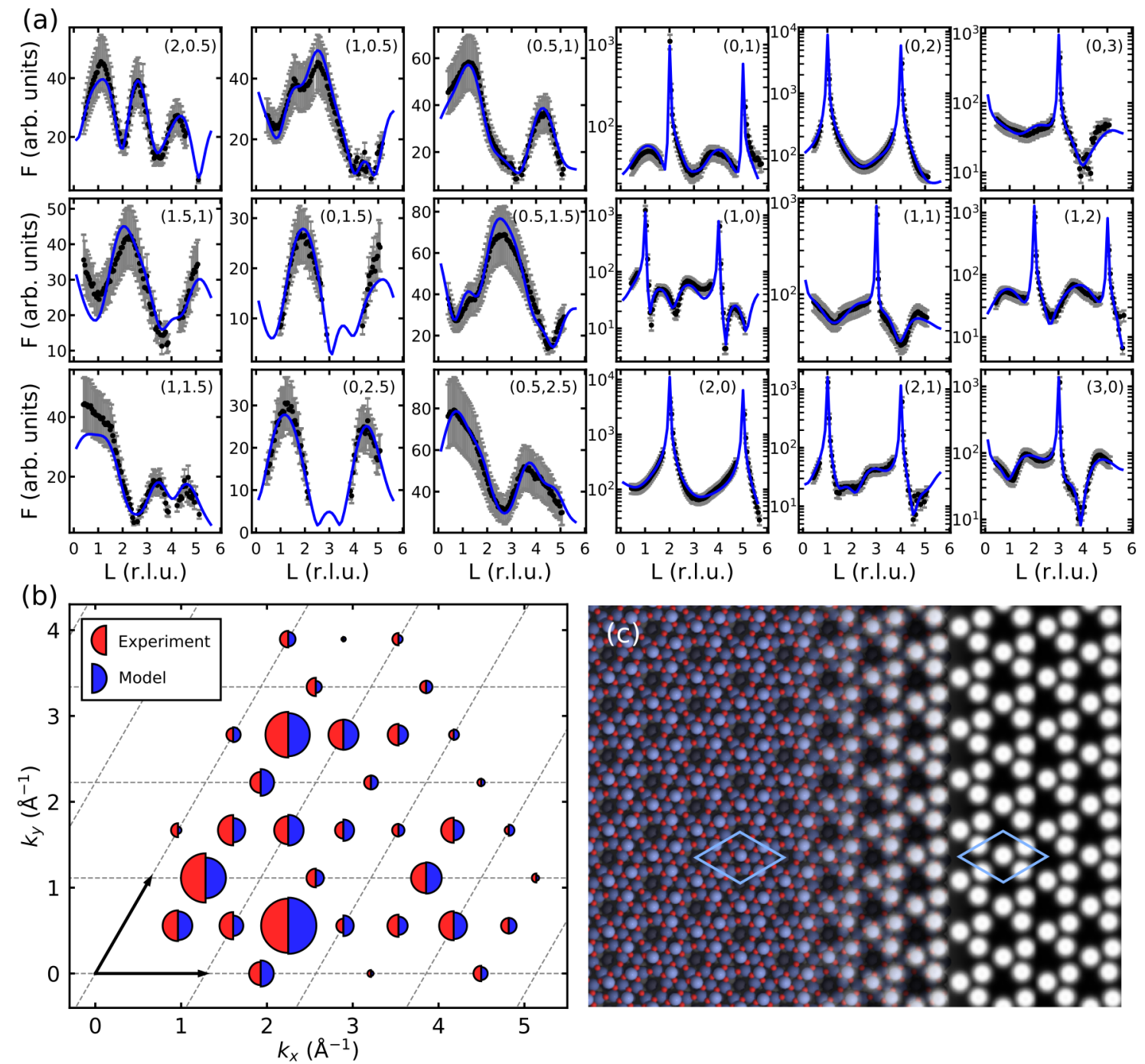

Figure 4: (a) Measured (black) and fitted (blue) X-ray diffraction rod profiles for the $(4 \times 4)$ phase. (b) Reciprocal space map showing the measured and calculated in-plane X-ray structure factors for the $(4 \times 4)$ phase. Dashed lines and arrows indicate the $(2 \times 2)$ unit cell of the ordered alloy surface used as a reference. (c) Simulated AFM frequency-shift image of the $\mathrm{Sn}_{11} \mathrm{O}_{12}$ structure, based on DFT calculations. ${ }^{32}$

geometry. The trigonal pyramidal geometry found here is nevertheless typical of $\mathrm{Sn}^{2+}$, as seen in halides and in tin(II) sulfide, and attributed to the occupation of a stereoactive lone pair orbital derived from the Sn5s state. This geometry has also been observed or predicted in oxidic phases, including layered, mixed-valence $\mathrm{Sn}_{3} \mathrm{O}_{4},{ }^{33}$ in polyoxometalate clusters, ${ }^{34}$ and in 
the surface layers of reduced $\mathrm{SnO}_{2}(101)^{35}$ and $\mathrm{SnO}_{2}(110) .^{20}$

Bonding between $\mathrm{Sn}$ in the oxide layer and $\mathrm{Pt}$ in the substrate is also typical of $\mathrm{Sn}^{2+}$. The strength of these bonds is reflected in outward buckling of surface Pt (opposite to the clean $\mathrm{Pt}_{3} \mathrm{Sn}(111)$ surface, where outward buckling of $\mathrm{Sn}$ is observed $\left.{ }^{36}\right)$, and in contracted Pt-Sn bond lengths similar to what is found in organometallic clusters. ${ }^{37,38}$ The solution of the structure of the well-ordered $(4 \times 4)$ phase thus yields the likely generalization that strong interfacial $\mathrm{Pt}-\mathrm{Sn}^{2+}$ bonding is a characteristic feature of $\mathrm{Pt} / \mathrm{SnO}$ interfaces. The particular structure found here results from optimization of these bonds at the (111) surface, constrained by preferred Sn-O bond geometry.

Although these structural features can be rationalized rather easily in hindsight, the structure itself could not be directly deduced from experiment or guessed based on chemical intuition, primarily due to its multi-layered arrangement and relatively low symmetry. An automated search method based on theory can, however, be used to generate physically plausible structural candidates for comparison with experiment, leading to the correct structure model. This methodology depends on a search algorithm that is both efficient and reliable. Our strategy utilizes the full history of the evolutionary search both to accelerate structure evaluation and to maintain a sufficiently diverse population so that premature convergence is avoided. With this we take a step towards optimal search algorithms in which all components leverage the available data to maximum benefit.

The $(4 \times 4)$ surface oxide on $\mathrm{Pt}_{3} \mathrm{Sn}(111)$ is relatively complex, but it nevertheless represents a rather ideal case, where quantitative diffraction measurements can be used to verify the result of the structure search. In general, surfaces can exhibit considerable degrees of disorder, with various coexisting phases and minority structures that cannot be characterized in detail by averaging techniques. The combination of scanning probe microscopy with theoretical simulations is often the only viable methodology in these cases, resulting in considerable uncertainty and occasionally gross misinterpretation. A reliable global optimization method like GOFEE can 
enable surface studies of this type to be conducted with much greater confidence.

Broad application of this approach, however, will also require the ability to characterize larger-scale features like defects and superstructures. For this, the search algorithms must be able to scale toward hundreds of atoms, resulting in drastically more demanding searches due to the combinatorial scaling of the configuration space and the increased cost of DFT evaluations. This will require even more effective data utilization in search strategies, where the efforts presented in this work, along with recent advances in transferable machine learning potentials ${ }^{39-41}$ and reinforcement learning protocols ${ }^{42}$ may pave the way for such scalable approaches. Avenues for continued progress in search methodologies are thus foreseen to ensure improved thoroughness and efficiency of automated structural search methods.

\section{Conclusions}

Surface structure determination is a notoriously difficult problem due to the limitations of experimental techniques and the high cost of accurate theoretical modeling. In this work we have demonstrated how a machine learning driven search algorithm can be used to overcome such limitations, through the characterization of an experimentally observed $(4 \times 4)$ surface oxide on $\mathrm{Pt}_{3} \mathrm{Sn}(111)$. For this application, we have extended a previously reported algorithm with a clustering-based strategy to maintain diversity in in search population which leverages the full history of structures visited in the search. The result is a structure prediction algorithm that is both robust and efficient, and represents a significant improvement over the traditional search method involving generation of trial structures manually based on intuition. This should increase the efficiency and reliability of surface studies in the future.

Acknowledgement: We acknowledge Diamond Light Source for time on Beamline I07 under Proposal SI17608. We are grateful to Hadeel Hussain and Jonathan Rawle for assistance with the surface X-ray diffraction measurements. We acknowledge MAX IV Laboratory for use of an STM instrument and thank Nikolay Vinogradov for assistance with these measurements. 
Henrik Grönbeck and Mattias Vandichel are acknowledged for helpful discussions. This work was performed as part of the Knut and Alice Wallenberg (KAW) funded project "Atomistic design of new catalysts" (Project No. KAW 2015.0058). Additional funding was provided by the Swedish Research Council through Grant Nos. 2018-05374 and 349-2011-6491. This work has been supported by the Danish National Research Foundation through the Center of Excellence "InterCat" (Grant agreement no.: DNRF150). MKB and BH acknowledge support from VILLUM FONDEN (Investigator grant, Project No. 16562). This research was supported by the Austrian Science Fund (FWF, SFB Project 'TACO', F81).

Author contributions: Conceptualization: LRM, MKB, UD, EL, BjH; Investigation: LRM, MKB, IS, MSe, BeH, MSh, MSch, EL; Methodology: MKB, BjH; Writing - original draft: LRM, MKB, IS, BjH; Writing - review and editing: LRM, MKB, IS, MSe, MSch, UD, EL, $\mathrm{BjH}$.

Competing interests: Authors declare that they have no competing interests.

Supplementary materials: Methods, Figs. S1 to S3, Table S1

\section{References}

[1] R. Burch, J. Catal. 1981, 71, $348-359$.

[2] R. D. Cortright, J. M. Hill, J. A. Dumesic, Catal. Today 2000, 55, 213-223.

[3] F. Coloma, A. Sepúlveda-Escribano, J. Fierro, F. Rodríguez-Reinoso, Appl. Catal. A 1996, $136,231-248$.

[4] A. Moscu, L. Veyre, C. Thieuleux, F. Meunier, Y. Schuurman, Catal. Today 2015, 258, 241-246. 
[5] M. Arenz, V. Stamenkovic, B. Blizanac, K. Mayrhofer, N. Markovic, P. Ross, J. Catal. 2005, 232, $402-410$.

[6] C. Lamy, S. Rousseau, E. Belgsir, C. Coutanceau, J.-M. Léger, Electrochim. Acta 2004, 49, 3901-3908.

[7] M. Batzill, D. E. Beck, B. E. Koel, Appl. Phys. Lett. 2001, 78, 2766-2768.

[8] M. Batzill, D. E. Beck, B. E. Koel, Phys. Rev. B 2001, 64, 245402.

[9] M. Batzill, J. Kim, D. E. Beck, B. E. Koel, Phys. Rev. B 2004, 69, 165403.

[10] A. Atrei, U. Bardi, G. Rovida, M. Torrini, M. Hoheisel, S. Speller, Surf. Sci. 2003, 526, 193-200.

[11] M. Hoheisel, S. Speller, W. Heiland, A. Atrei, U. Bardi, G. Rovida, Phys. Rev. B 2002, 66, 165416.

[12] J. Zheng, M. Busch, L. Artiglia, T. Skála, J. Rossmeisl, S. Agnoli, Adv. Mater. Interfaces 2019, 6, 1801874.

[13] C. J. Pickard, R. J. Needs, J. Phys.: Condens. Matter 2011, 23, 053201.

[14] A. Oganov, C. Pickard, Q. Zhu, R. Needs, Nat. Rev. Mater. 2019, 4, 331-348.

[15] A. R. Oganov, C. W. Glass, J. Chem. Phys. 2006, 124, 244704.

[16] Y. Wang, J. Lv, L. Zhū, Y. ma, Phys. Rev. B 2010, 82, 094116.

[17] L. B. Vilhelmsen, B. Hammer, J. Chem. Phys. 2014, 141, 044711.

[18] S. Woodley, G. Day, R. Catlow, Phil. Trans. R. Soc. A 2020, 378, 20190600.

[19] M. Sierka, T. K. Todorova, J. Sauer, S. Kaya, D. Stacchiola, J. Weissenrieder, S. Shaikhutdinov, H.-J. Freund, J. Chem. Phys. 2007, 126, 234710. 
[20] L. R. Merte, M. S. Jørgensen, K. Pussi, J. Gustafson, M. Shipilin, A. Schaefer, C. Zhang, J. Rawle, C. Nicklin, G. Thornton, R. Lindsay, B. Hammer, E. Lundgren, Phys. Rev. Lett. 2017, 119, 096102.

[21] H. Zakaryan, A. Kvashnin, A. Oganov, Sci. Rep. 2017, 7, 10357.

[22] V. Deringer, D. Proserpio, G. Csanyi, C. Pickard, Faraday Discuss. 2018, 211, 45-59.

[23] Q. Tong, L. Xue, J. Lv, Y. Wang, Y. Ma, Faraday Discuss. 2018, 211, 31-43.

[24] M. K. Bisbo, B. Hammer, Phys. Rev. Lett. 2020, 124, 086102.

[25] M. S. Jørgensen, M. N. Groves, B. Hammer, J. Chem. Theory Comput. 2017, 13, 14861493.

[26] Y. Wang, C. Dang, H. Li, L. Han, J. Wei in 2009 IEEE Congress on Evolutionary Computation, pp. 2927-2933.

[27] Y. Yuan, H. Xu, B. Wang, X. Yao, IEEE Trans. Evol-Comput. 2016, 20, 16-37.

[28] X. Zhang, Y. Tian, R. Cheng, Y. Jin, IEEE Trans. Evol. Comput. 2018, 22, 97-112.

[29] V. R. Stamenković, M. Arenz, C. A. Lucas, M. E. Gallagher, P. N. Ross, N. M. Marković, J. Am. Chem. Soc. 2003, 125, 2736-2745.

[30] D. Arthur, S. Vassilvitskii in Proceedings of the Eighteenth Annual ACM-SIAM Symposium on Discrete Algorithms, Society for Industrial and Applied Mathematics, USA, of SODA '07, p. 1027-1035.

[31] We note that this approach deviates from the definition of a population in the biological sense, but we choose to retain this term, as it serves the same purpose in the search. 
[32] I. Sokolović, M. Reticcioli, M. Čalkovský, M. Wagner, M. Schmid, C. Franchini, U. Diebold, M. Setvín, Proc. Natl. Acad. Sci. 2020, 117, 14827-14837.

[33] A. Seko, A. Togo, F. Oba, I. Tanaka, Phys. Rev. Lett. 2008, 100, 045702.

[34] K. Suzuki, T. Hanaya, R. Sato, T. Minato, K. Yamaguchi, N. Mizuno, Chem. Commun. 2016, 52, 10688-10691.

[35] M. Batzill, A. M. Chaka, U. Diebold, Europhys. Lett. 2004, 65, 61-67.

[36] A. Atrei, U. Bardi, G. Rovida, M. Torrini, E. Zanazzi, P. N. Ross, Phys. Rev. B 1992, 46, $1649-1654$.

[37] M. Bortoluzzi, A. Ceriotti, I. Ciabatti, R. Della Pergola, C. Femoni, M. Carmela Iapalucci, A. Storione, S. Zacchini, Dalton Trans. 2016, 45, 5001-5013.

[38] M. C. Jennings, G. Schoettel, S. Roy, R. J. Puddephatt, Organometallics 1991, 10, 580586.

[39] V. L. Deringer, N. Bernstein, G. Csányi, C. Ben Mahmoud, M. Ceriotti, M. Wilson, D. A. Drabold, S. R. Elliott, Nature 2021, 589, 59-64.

[40] J. S. Smith, B. T. Nebgen, R. Zubatyuk, N. Lubbers, C. Devereux, K. Barros, S. Tretiak, O. Isayev, A. E. Roitberg, Nat. Commun. 2019, 10, 2903.

[41] C. van der Oord, G. Dusson, G. Csányi, C. Ortner, Mach. Learn.: Sci. Technol. 2020, 1, 015004.

[42] H. L. Mortensen, S. A. Meldgaard, M. K. Bisbo, M.-P. V. Christiansen, B. Hammer, Phys. Rev. B 2020, 102, 075427. 